\title{
Low-energy electron scattering from methanol and ethanol
}

\author{
M. A. Khakoo, ${ }^{1}$ J. Blumer, ${ }^{2}$ K. Keane, ${ }^{1}$ C. Campbell, ${ }^{1}$ H. Silva, ${ }^{3}$ M. C. A. Lopes, ${ }^{3}$ C. Winstead, ${ }^{4}$ V. McKoy, ${ }^{4}$ \\ R. F. da Costa, ${ }^{5}$ L. G. Ferreira, ${ }^{6}$ M. A. P. Lima, ${ }^{5}$ and M. H. F. Bettega ${ }^{7}$ \\ ${ }^{1}$ Department of Physics, California State University, Fullerton, California 92831, USA \\ ${ }^{2}$ Department of Mechanical Engineering, Brigham Young University, Provo, Utah 84602, USA \\ ${ }^{3}$ Departamento de Física, ICE, Universidade Federal de Juiz de Fora, Juiz de Fora-MG, CEP 36036-330, Brazil \\ ${ }^{4}$ A. A. Noyes Laboratory of Chemical Physics, California Institute of Technology, Pasadena, California 91125, USA \\ 5 Instituto de Física "Gleb Wataghin," Universidade Estadual de Campinas, Caixa Postal 6165, 13083-970, Campinas, São Paulo, Brazil \\ ${ }^{6}$ Instituto de Física, Universidade de São Paulo, Caixa Postal 66318, 05315-970, São Paulo, São Paulo, Brazil \\ ${ }^{7}$ Departamento de Física, Universidade Federal do Paraná, Caixa Postal 19044, 81531-990, Curitiba, Paraná, Brazil
}

(Received 23 January 2008; published 9 April 2008)

\begin{abstract}
Measured and calculated differential cross sections for elastic (rotationally unresolved) electron scattering from two primary alcohols, methanol $\left(\mathrm{CH}_{3} \mathrm{OH}\right)$ and ethanol $\left(\mathrm{C}_{2} \mathrm{H}_{5} \mathrm{OH}\right)$, are reported. The measurements are obtained using the relative flow method with helium as the standard gas and a thin aperture as the collimating target gas source. The relative flow method is applied without the restriction imposed by the relative flow pressure conditions on helium and the unknown gas. The experimental data were taken at incident electron energies of $1,2,5,10,15,20,30,50$, and $100 \mathrm{eV}$ and for scattering angles of $5^{\circ}-130^{\circ}$. There are no previous reports of experimental electron scattering differential cross sections for $\mathrm{CH}_{3} \mathrm{OH}$ and $\mathrm{C}_{2} \mathrm{H}_{5} \mathrm{OH}$ in the literature. The calculated differential cross sections are obtained using two different implementations of the Schwinger multichannel method, one that takes all electrons into account and is adapted for parallel computers, and another that uses pseudopotentials and considers only the valence electrons. Comparison between theory and experiment shows that theory is able to describe low-energy electron scattering from these polyatomic targets quite well.
\end{abstract}

DOI: 10.1103/PhysRevA.77.042705

PACS number(s): $34.80 . \mathrm{Bm}$

\section{INTRODUCTION}

Low-energy electron collision processes play an important role in several areas including plasmas [1-3], astrophysics [4], and radiation biology [5]; electron-alcohol collision processes, in particular, are relevant to modeling spark ignition in alcohol-fueled internal combustion engines [6,7]. Elastic collisions are also a dominant process in low-energy electron transport through gaseous media and condensed matter, and are thus important in our understanding of the transport of electrons in media such as organic tissue [8], planetary atmospheres [4], interstellar media, lasers, and fusion plasmas [9].

The seminal work of Boudaiffa et al. [5] demonstrating DNA strand breakage by low-energy electrons has simulated renewed interest in the role of electron collisions in biological processes. Bouchiha et al. [10] have carried out $R$-matrix calculations for low-energy electron scattering by methanol, continuing a program [11] of studies of electron interactions with prototypical organic molecules to obtain insights regarding biomolecules. Similar studies, also addressing larger biomolecules, have been undertaken by Tonzani and Greene $[12,13]$ using a grid-based $R$-matrix code and by Winstead and McKoy [14-17] using the Schwinger multichannel method. Bettega [18] has carried out a study of low energy electron collisions with formic acid, the simplest organic acid. Shape resonances in electron collisions with furan, a system similar to tetrahydrofuran, have also been studied by Bettega and Lima [19], and the influence of polarization on the electron-impact excitation cross sections for this system has been investigated by da Costa, Bettega, and Lima [20].
Electron transmission spectra of methanol have been studied by Mathur and Hasted [21], while total electron scattering cross-section measurements have been made by Schmieder [22], by Sueoka et al. [23], and most recently by Szmytkowski and Krzysztofowicz [24]. Measurements of resonant dissociative electron attachment to methanol have been reported by von Trepka and Neuert [25], by Kühn et al. [26], by Curtis and Walker [27], by Prabhudesai et al. [28], by Skalický and Allan [29], and, in the condensed phase, by Parenteau et al. [30]. Skalický and Allan [29] also assigned grandparent states to the resonances observed in their attachment spectra using He I photoelectron spectroscopy. Ibănescu et al. [31] extended the work of Skalický and Allan [29], observing a new peak at $2.99 \mathrm{eV}$ in the $\mathrm{CH}_{3} \mathrm{O}^{-}$yield which they assigned to an $\mathrm{O}-\mathrm{H} \sigma^{*}$ resonance. Wen et al. [32] have measured electron-impact vibrational excitation cross sections for condensed-phase methanol and observed several broad resonances. Ethanol has been less well studied than methanol. Dissociative attachment spectra revealing a variety of resonance peaks were recently reported by Prabhudesai $e t$ al. [28], Ibănescu et al. [31], and Orzol et al. [33]; the second group also measured vibrational excitation cross sections and photoelectron spectra. The latter two groups observed a lowenergy peak in the yield of $\mathrm{C}_{2} \mathrm{H}_{5} \mathrm{O}^{-}$similar to the $\mathrm{CH}_{3} \mathrm{O}^{-}$ peak seen in methanol [31], albeit the spectrum of Orzol et al. [33] appears shifted to lower energy relative to that of Ibănescu et al. [31].

To our knowledge there have been no measurements of elastic electron scattering cross sections of methanol or ethanol. This is partly because, to date, measurements of elastic electron scattering differential cross sections (DCSs) for gaseous targets have mainly employed the conventional relative 
flow method, which was initially devised and applied to $\mathrm{N}_{2}$ by Srivastava et al. [34] in 1975 and is now the main method in use for quantitative measurements of DCSs. Details of this method can be found in Trajmar et al. [35] and Brunger and Buckman [1]. The method relies on a comparison of the electron scattering signal of the unknown gas $X$ with that of $\mathrm{He}$, whose DCSs are accurately known either from measurements or calculations (see, for example, Nesbet [36] or Register et al. [37]). However, the relative flow method (using conventional tube collimating gas sources) has been limited to targets whose gas-kinetic cross sections are known or can be accurately estimated, and thus has been largely restricted to atoms such as $\mathrm{He}$ and the noble gases, diatomic molecules such as $\mathrm{H}_{2}, \mathrm{~N}_{2}, \mathrm{O}_{2}$, and $\mathrm{CO}$, and small polyatomic molecules such as $\mathrm{CO}_{2}, \mathrm{CH}_{4}, \mathrm{C}_{2} \mathrm{H}_{2}$, and $\mathrm{C}_{2} \mathrm{H}_{4}$. There have, however, been recent quantitative measurements of electron scattering from polyatomic targets such as tetrahydrofuran (THF), whose elastic electron scattering has been studied by Milosavljević et al. [38], Dampc et al. [39], Colyer et al. [40], and Allan [41], employing the relative flow technique and a molecular diameter value from [42]. Though agreement between the DCSs of Allan [41] and Colyer et al. [40] is very good, there are significant disagreements, in some cases by as much as a factor of 2, between the data of Dampc et al. [39] and Milosavljević et al. [38].

This paper extends our recent implementation of the relative flow method [43], which does not require gas-kinetic molecular diameters, to methanol and ethanol. The method is a simple modification of the conventional relative flow method in which a collimating tube source is replaced by an aperture source and has been tested with $\mathrm{N}_{2}$ and $\mathrm{C}_{2} \mathrm{H}_{4}$ using $\mathrm{He}$ as a calibration standard [43]. In addition to the measurements, we present results of calculations using the Schwinger multichannel (SMC) method. These calculations were carried out using two different implementations of the SMC method. One set of calculations employed a parallel implementation of the SMC method that takes all electrons into account [44] while the other calculations used normconserving pseudopotentials and explicitly included only the valence electrons [45]. Both studies were carried out in the fixed-nuclei static-exchange (SE) and static-exchange-pluspolarization (SEP) approximations and therefore correspond to rotationally and vibrationally unresolved, electronically elastic results.

\section{METHOD}

\section{A. Experiment: Aperture-source relative flow method}

The relative flow method of Srivastava et al. [34], a successful and very popular procedure for measuring differential elastic electron scattering by light atoms and molecules, has been applied extensively in the last 30 years to determine elastic electron scattering DCSs for many such targets. The review by Brunger and Buckman [1] provides an excellent summary of this work. Details of the apparatus used in the present study (spectrometer, vacuum chamber, control equipment) can be found elsewhere [46] and only a brief description will be given here. Cylindrical electrostatic optics and double hemispherical energy selectors were utilized both in the electron gun and in the detector. Energy loss spectra of the elastic peak were collected at fixed incident energies and scattering angles by repetitive, multichannel-scaling techniques. The target gas beam was formed by effusing the gas through an aperture which was constructed by mounting a disk of thin $(0.025 \mathrm{~mm})$ brass shim stock into a flush recess at the end of a brass tube $(6.35 \mathrm{~mm}$ o.d. and $4.3 \mathrm{~mm}$ i.d.) and punching the aperture $(0.3 \mathrm{~mm}$ diameter $)$ into the shim stock with a straight sharp sewing needle while the brass tube was rotating in the lathe. The aperture was thus located centrally and flush with the end of the tube. The tube was incorporated into a moveable source [47] arrangement. The moveable gas source method has been well-tested previously in our laboratory $[48,49]$ and determines background scattering rates expediently and accurately in electron scattering experiments. In this method, the collimating gas structure is moved into (signal+background) and out of (background) the collision region center. Following the procedure of Ref. [50] (see also [43]) and employing the moveable gas source method:

(i) We calibrated the behavior of our gas-handling system for $\mathrm{He}, \mathrm{CH}_{3} \mathrm{OH}$, and $\mathrm{C}_{2} \mathrm{H}_{5} \mathrm{OH}$, for the relative flow rate, $\mathcal{R}$, vs the drive source pressure, $P_{s}$, under steady state conditions for all three gases. The liquids were housed in a flask vacuum coupled to the leak valves used to send the vapor into the gas feed line. American Chemical Society (ACS) grade ( $>99.94 \%$ purity) $\mathrm{CH}_{3} \mathrm{OH}$ and $\mathrm{C}_{2} \mathrm{H}_{5} \mathrm{OH}$ and high purity He ( $>99.995 \%$ purity) were used. The copper gas feed lines (made from standard refrigeration tubing) were held at the elevated temperature (relative to room temperature) of $320 \mathrm{~K}$ to prevent condensation in the feed lines. That there was no condensation in these lines was established by reproducing flow rates with increasing and decreasing source drive pressures. To prevent the vapor from condensing in the spectrometer, the spectrometer was heated to about $393 \mathrm{~K}$. This heating stabilized the spectrometer and made scattering rates reproducible, whereas at lower temperatures the analyzer surfaces were affected by the vapor. However, despite this heating, there was condensation of alcohol vapor on the outer walls of our vacuum chamber (which were at room temperature of $297 \mathrm{~K}$ ), resulting in a slow fall off of the chamber pressure when either the $\mathrm{CH}_{3} \mathrm{OH}$ or $\mathrm{C}_{2} \mathrm{H}_{5} \mathrm{OH}$ was shut off, in contrast to He. However, this did not affect in any way the reproducibility of our DCSs or the stability of the electron beam. A second-order polynomial in $P_{s}$ served adequately to express $\mathcal{R}$ as a function of $P_{s}$ in the form $\mathcal{R}$ $=a P_{s}\left(1+\varepsilon P_{s}\right)$, as suggested by Sagara and Boesten [51] and discussed in [52]. The coefficients $a$ and $\varepsilon$ are related to the molar masses (M) and the molecular diameters $\delta$ of the gases (relative to HE of $2.18 \times 10^{-8} \mathrm{~cm}$ ) and are given in Table I, which also includes these values for propanol (on-going work). The gas-kinetic molecular diameters determined from our flow measurements for the alcohols are seen to be large compared to nonpolar hard-sphere molecules and reflect the long-range dipole forces between these polar molecules. However, the $a$ coefficient for these molecules deviates markedly, by a factor of $\sim 2$, from its molecular mass dependence (last column in Table I, mass factor). The reason for this is not clear, but these molecules could possibly form dimers during their flow through the gas system from the liquid phase into the gas phase. Though many polar gases 
TABLE I. Gas flow parameters for the aperture source for various gases. See text for discussion.

\begin{tabular}{|c|c|c|c|c|c|c|c|c|}
\hline Gas & $\begin{array}{c}T \\
\left({ }^{\circ} \mathrm{C}\right)\end{array}$ & $\begin{array}{c}a \\
\left(\mathrm{~s}^{-1}\right)\end{array}$ & $\begin{array}{c}\varepsilon \\
\left(\text { Torr }^{-1}\right)\end{array}$ & $\delta\left(10^{-8} \mathrm{~cm}\right)$ & M & $a \sqrt{M} \sqrt{M_{f}}$ & $\varepsilon / \delta^{2}$ & $M_{f}$ \\
\hline $\mathrm{H}_{2}$ & 25 & 0.2181 & 1.050 & 2.74 & 2.016 & 0.310 & 1.40 & 1 \\
\hline $\mathrm{He}$ & 25 & 0.1551 & 0.739 & 2.18 & 4.002 & 0.310 & 1.56 & 1 \\
\hline $\mathrm{He}$ & 47 & 0.1611 & 0.638 & 2.18 & 4.002 & 0.322 & 1.34 & 1 \\
\hline $\mathrm{He}$ & 74 & 0.1615 & 0.659 & 2.18 & 4.002 & 0.323 & 1.39 & 1 \\
\hline $\mathrm{N}_{2}$ & 25 & 0.0596 & 2.115 & 3.75 & 28.02 & 0.316 & 1.50 & 1 \\
\hline $\mathrm{C}_{2} \mathrm{H}_{4}$ & 25 & 0.0566 & 4.070 & 4.95 & 28.03 & 0.300 & 1.66 & 1 \\
\hline $\mathrm{CH}_{3} \mathrm{OH}$ & 47 & 0.0387 & 5.722 & 6.30 & 32.04 & 0.310 & 1.44 & 2 \\
\hline $\mathrm{CH}_{3} \mathrm{OH}$ & 74 & 0.0475 & 4.614 & 5.67 & 32.04 & 0.329 & 1.44 & 1.5 \\
\hline $\mathrm{C}_{2} \mathrm{H}_{5} \mathrm{OH}$ & 47 & 0.0352 & 7.346 & 7.15 & 46.07 & 0.337 & 1.44 & 2 \\
\hline $\mathrm{C}_{2} \mathrm{H}_{5} \mathrm{OH}$ & 74 & 0.0373 & 6.508 & 6.73 & 46.07 & 0.339 & 1.44 & 1.8 \\
\hline $\mathrm{C}_{3} \mathrm{H}_{7} \mathrm{OH}$ & 74 & 0.0288 & 8.095 & 7.49 & 60.11 & 0.316 & 1.44 & 2 \\
\hline $\mathrm{C}_{4} \mathrm{H}_{9} \mathrm{OH}$ & 74 & 0.0212 & 9.754 & 8.23 & 74.12 & 0.327 & 1.44 & 3.2 \\
\hline
\end{tabular}

form dimers when compressed, any alcohol dimers will break up rapidly under the vacuum and elevated temperature conditions in the gas flow lines.

Another issue is the sticking of these polar gases on surfaces during their flow through the gas handling system, which was addressed by Allan [41]. As in THF, in this system we observed a linear dependence of the pressure with time when the relative flow was determined using a closed volume (see [43]). Sticking would, in principle, cause the pressure versus time dependence to be proportional to pressure and thus to become nonlinear. It would also result in hysteresis in the determination of pressure versus flow rate, i.e., different curves depending on whether the pressure was being increased or decreased. Surface sticking also does not explain the consistent factor of 2 (see previous paragraph) for all the alcohols studied. One would expect sticking to be dependent on the heat of adsorption (which would vary among the alcohols studied here including propanol) and also make it difficult to precisely reproduce the DCSs for different flow conditions.

(ii) We established the incident energy of our electron beam to within an estimated $\pm 0.04 \mathrm{eV}$ at the start of the experiment by correcting it for the contact potential of the spectrometer using the $1 s 2 s^{2}\left({ }^{2} S\right)$ resonance in $\mathrm{He}$ at the incident energy of $19.366 \mathrm{eV}$ [53]. This contact potential remained stable over several days, but was monitored repeatedly on a daily basis.

(iii) We conducted our experiment at low pressures (typically $<2$ Torr for $\mathrm{He},<0.2$ Torr for $\mathrm{CH}_{3} \mathrm{OH}$, and $<0.12$ for $\mathrm{C}_{2} \mathrm{H}_{5} \mathrm{OH}$ ) where the mean free path is well below the critical mean free path (equal to the aperture thickness of $0.025 \mathrm{~mm}$ ) conditions for these gases, which occur at pressures of 5 Torr for $\mathrm{He}, \sim 0.6$ Torr for $\mathrm{CH}_{3} \mathrm{OH}$, and $<0.45$ Torr for $\mathrm{C}_{2} \mathrm{H}_{5} \mathrm{OH}$. At $1 \mathrm{eV}$ and $2 \mathrm{eV}$, He was operated at a slightly higher pressure $(\approx 2.5$ Torr) because the low-angle DCSs in $\mathrm{He}$ are small and higher pressure enabled us to accumulate enough signal in the standard acquisition time allotted to the other gases. This was done to maintain the stability of the electron beam to better than $10 \%$ during the experiment. The pressure in the experimental chamber was typically in the range of $(0.7-2) \times 10^{-6}$ Torr and the incident electron current remained stable during operation to within $10 \%$ at most. Interestingly, the effect of energy broadening in elastic collisions with $\mathrm{He}$ (the lighter target) cannot be fully ignored in the overall assessment of the DCSs. A rough calculation shows that for our cosine beam, it would broaden the elastic peak by an additional $3-6 \mathrm{meV}$ for a 1 to $2 \mathrm{~mm}$ interaction region placed $6 \mathrm{~mm}$ downstream from the aperture. Experimentally, this was checked by comparing the elastic spectral feature for He against, for example, propanol or methanol. The elastic peak's energy profile [full width at half maximum (FWHM)] did not change (at least observably) from the 50-60 meV FWHM when different gases flowed through the aperture. We note that the present spectrometer's energy resolution does not resolve rotational structure in the elastic peak, hence the present DCSs are rotationally unresolved for elastic scattering.

(iv) Our spectrometer acquisition was mostly computercontrolled. The computer located the scattering angle and scanned the elastic scattering spectra, storing these along with the sum of the pressures (taken for each scan) in the Baratron manometer $\left(=\Sigma_{n} P_{s} ; n\right.$ is the number of scans) along with the squared-sum of the pressures $\left(=\Sigma_{n} P_{s}^{2}\right)$, from which the average pressure, $\bar{P}_{s}\left(=\Sigma_{n} P_{s} / n\right)$, and its standard deviation error-squared, $\sigma\left(P_{s}\right)^{2}=\frac{1}{n-1}\left[\Sigma_{n} P_{s}^{2}-n \bar{P}_{s}^{2}\right]$, can be computed and used for error estimation. With these values, $\mathcal{R}$ and its error could be computed from the polynomial expression for $\mathcal{R}$ vs $P_{s}$ described in (i). The spectrometer covered the angles and scans (with equal times with gas aligned with the electron beam, and gas away from the electron beam) as prescribed in a file of these angles. The relative electron current was obtained from a sooted molybdenum beam flag placed in front of the electron beam with an approximately $35 \mathrm{~V}$ bias relative to ground to collect the incident electrons. The flag, with bias on, was used during every spectrometerangle changing interval to measure the incident current and was then moved out of place, and its bias set to ground (collision region potential). A constant in-line electron current measurement device was not used here so as not to introduce further secondary electrons that emerge from Far- 
aday cup devices in general. The gas whose cross section was to be determined was flowed following $\mathrm{He}$, scattered count rates for a range of angles were obtained, and the process was then repeated to check for reproducibility. In all cases the electron beam did not change by more than $10 \%$ for different gases.

(v) The differential cross section for either methanol or ethanol $\left[\sigma_{X}\left(E_{0}, \theta\right)\right]$ at the incident electron energy $E_{0}$ and scattering angle $\theta$ was then obtained from the relative flow equation $[1,43]$ :

$$
\sigma_{x}\left(E_{0}, \theta\right)=\sigma_{\mathrm{He}}\left(E_{0}, \theta\right) \frac{\mathcal{R}_{\mathrm{He}}}{\mathcal{R}_{X}} \frac{\mathcal{S}_{X}}{\mathcal{S}_{\mathrm{He}}} \sqrt{\frac{M_{\mathrm{He}}}{M_{X}}},
$$

where $\mathcal{S}$ is the background-corrected scattering rate and subscripts " $X$ " $\left(\mathrm{CH}_{3} \mathrm{OH}\right.$ or $\left.\mathrm{C}_{2} \mathrm{H}_{5} \mathrm{OH}\right)$ and "He" indicate the gases used. $M$ is the molar mass and the He elastic DCSs were taken from [36,37].

We measured elastic scattering cross sections for $\mathrm{CH}_{3} \mathrm{OH}$ and $\mathrm{C}_{2} \mathrm{H}_{5} \mathrm{OH}$ at $1,2,5,10,15,20,30,50$, and $100 \mathrm{eV}$ at scattering angles from $5^{\circ}$ to $130^{\circ}$, with a coarser interval $\left(20^{\circ}\right)$ at large scattering angles than at small scattering angles $\left(10^{\circ}\right)$. We note in particular that at $E_{0}=1 \mathrm{eV}$, which is the lower limit to our instrument, the small angle signal in $\mathrm{He}$ was most difficult to establish because of the low elastic scattering DCSs for $\mathrm{He}$ at low energies; our error bars are thus largest at $1 \mathrm{eV}$.

Our DCSs were integrated to obtain integral cross sections (ICS) by extrapolating the forward and backward experimental DCSs, estimating the form of these DCSs at angles below and above the measured range using theoretical DCSs as a guide. However, the Born method leads to very large forward DCSs, so we extrapolated our experimental DCSs to lower values. We note that in the solid-angle integration of the DCSs, the $\sin \theta$ term reduces the effect of the small and large angle DCSs, weighting the $\theta=90^{\circ}$ DCSs the most. To gauge the precision of our integration we set the small and large angle DCSs equal to that of our smallest angle DCS and the largest $\left(130^{\circ}\right)$ angle DCS (flat extrapolation). The difference between the flat-extrapolated integration and the estimated extrapolation was incorporated into our total ICS errors and usually did not exceed $15 \%$. These extrapolated DCSs used in our determination of experimental ICSs are given in Table II. This (standard) method is reliable and has been used accurately in the past.

\section{B. Theoretical methods}

The calculations employed the SMC method. Two different implementations of this method were used in these calculations: one that takes all electrons into account and is adapted to parallel computers and another (SMCPP) that uses pseudopotentials to replace the core electrons and explicitly includes only the valence electrons. The SMC method and the above implementations have been described in detail elsewhere, and here we only present the details of the present calculations.

The calculations using the SMCPP method were carried out in the static-exchange (SE) and static-exchange plus polarization (SEP) approximations within the $C_{s}$ group. We used the equilibrium ground state geometry [54] in the bound state and scattering calculations. The basis used was generated by a variational procedure [55] and is the same as that used in [18]. This basis included $(5 s 5 p 3 d)$ functions on carbon and oxygen and $(3 s 1 p)$ on hydrogen. For methanol we also included additional functions $(3 s 3 p 2 d)$ at the center of mass, according to [18]. Polarization effects are accounted for by including in the trial configuration space antisymmetrized products of singly excited target configurations with one-particle scattering orbitals. This procedure is not unique unless we use the complete set of single excitations and scattering orbitals for the chosen Cartesian Gaussian set. When less than a complete set of configurations and orbitals is used, as must frequently be the case for practical reasons, the quality of the calculation will depend on how well the polarization space represents the important real and virtual excitations of the target, which in turn is influenced by the choice of virtual orbitals. For this reason, we tested different approaches for methanol before doing the ethanol calculation. For instance, the empty (particle) orbitals can be improved virtual orbitals (IVO) [56], modified virtual orbitals (MVO) [57], or polarized orbitals [58]. One must also choose whether to carry out calculations using only singlet-coupled excitations of the target or both singlet- and triplet-coupled excitations. Although not so important for the long-range character of the polarization potential, the triplet states can be essential for the short range description of polarization, especially for incident electron energies around resonance positions and for molecules with low-lying triplet states. With this in mind, we carried out different calculations for methanol, using MVOs and IVOs with different coupling schemes (only singlets and singlets plus triplets). The results from these different approaches were essentially the same and we choose to present for methanol results obtained from calculations that employed IVOs and singlets only. For methanol we included only singlet-coupled single excitations from the occupied orbitals to IVOs with energy less than 0.5 Hartree and used all IVOs as scattering orbitals. The entire calculation for methanol included 5426 doublet configuration state functions for $A^{\prime}$ symmetry and 4738 for $A^{\prime \prime}$ symmetry. The symmetric $3 s$ combinations of Gaussian $d$ functions $\left[\left(x^{2}+y^{2}+z^{2}\right) \exp \left(-\alpha r^{2}\right)\right]$ were not included in the basis. Our calculated dipole moment of $1.81 \mathrm{D}$ compares well with the experimental value of $1.7 \mathrm{D}$ [54].

For ethanol we used the same Cartesian Gaussian basis sets on each atom as in methanol (but with no extra functions at the center of mass) and followed a different strategy for the orbital basis in which the MVOs were constructed for the +2 ion formed by removing the electrons from the outermost occupied orbital. We included all singly excited configurations formed from MVOs with eigenvalues below $10 \mathrm{eV}$. This (particle) orbital space was also used as the scattering basis in the construction of the closed-channel trial configurations. The calculation for ethanol included 5532 doublet configuration state functions (constructed from antisymmetrized products of singlet- and triplet-coupled single excitations of the target and one-particle scattering functions) for the $A^{\prime}$ symmetry and 5255 for the $A^{\prime \prime}$ symmetry. The calculated dipole moment of $1.82 \mathrm{D}$ agrees well with the experimental value of $1.69 \mathrm{D}$ [59]. As for methanol, we employed 
TABLE II. Present experimental DCS and ICS values for elastic electron scattering from methanol and ethanol with 1 standard deviation errors. Units are in $10^{-16} \mathrm{~cm}^{2} \mathrm{sr}^{-1}$. Values in italics are extrapolated or interpolated DCSs used to obtain the ICSs quoted here.

$\mathrm{E}_{0} \rightarrow$

$\theta(\mathrm{deg}) \downarrow 1 \mathrm{eV}$ Error $2 \mathrm{eV}$ Error $5 \mathrm{eV}$ Error $10 \mathrm{eV}$ Error $15 \mathrm{eV}$ Error $20 \mathrm{eV}$ Error $30 \mathrm{eV}$ Error $50 \mathrm{eV}$ Error $100 \mathrm{eV}$ Error

\begin{tabular}{|c|c|c|c|c|c|c|c|c|c|c|c|c|c|c|c|c|c|c|}
\hline \multicolumn{19}{|c|}{ (a) Methanol } \\
\hline 0 & 35.0 & & 80.0 & & 100 & & 30.0 & & 40.0 & & 45.0 & & 60.0 & & 100 & & 100 & \\
\hline 5 & 30.0 & & 60.0 & & 70.0 & & 23.0 & & 30.0 & & 30.0 & & 35.0 & & 54.6 & 9.2 & 38.3 & 5.9 \\
\hline 10 & 25.0 & & 40.0 & & 40.0 & & 18.0 & 2.6 & 19.2 & 2.5 & 20.1 & 3.4 & 20.0 & 3.2 & 24.8 & 3.8 & 18.1 & 2.8 \\
\hline 15 & 20.0 & & 20.0 & & 18.5 & 3.3 & 14.0 & & 15.0 & & 14.5 & & 13.0 & & 12.0 & & 9.0 & \\
\hline 20 & 15.4 & 3.3 & 12.1 & 0.9 & 10.8 & 1.8 & 10.1 & 1.4 & 10.9 & 1.4 & 10.2 & 1.6 & 8.17 & 1.10 & 7.12 & 1.05 & 3.20 & 0.45 \\
\hline 30 & 9.85 & 1.65 & 5.45 & 0.67 & 5.32 & 0.80 & 6.08 & 0.81 & 5.56 & 0.68 & 4.34 & 0.65 & 3.13 & 0.43 & 1.63 & 0.25 & 0.841 & 0.112 \\
\hline 40 & 5.02 & 0.78 & 3.35 & 0.44 & 3.16 & 0.41 & 3.42 & 0.49 & 2.83 & 0.38 & 2.05 & 0.28 & 1.42 & 0.18 & 1.02 & 0.14 & 0.431 & 0.069 \\
\hline 50 & 3.34 & 0.56 & 2.24 & 0.29 & 2.22 & 0.31 & 2.04 & 0.28 & 1.75 & 0.22 & 1.29 & 0.20 & 0.932 & 0.120 & 0.533 & 0.077 & 0.266 & 0.035 \\
\hline 70 & 1.76 & 0.24 & 1.45 & 0.20 & 1.94 & 0.24 & 1.14 & 0.16 & 0.978 & 0.129 & 0.750 & 0.117 & 0.475 & 0.073 & 0.222 & 0.033 & 0.105 & 0.016 \\
\hline 90 & 1.19 & 0.16 & 1.50 & 0.18 & 1.89 & 0.24 & 1.21 & 0.17 & 0.709 & 0.098 & 0.525 & 0.089 & 0.271 & 0.041 & 0.142 & 0.021 & 0.065 & 0.009 \\
\hline 110 & 1.38 & 0.21 & 1.51 & 0.18 & 1.53 & 0.22 & 1.43 & 0.19 & 0.839 & 0.112 & 0.546 & 0.076 & 0.313 & 0.045 & 0.178 & 0.025 & 0.088 & 0.012 \\
\hline 130 & 1.87 & 0.26 & 1.18 & 0.16 & 1.30 & 0.18 & 1.47 & 0.20 & 1.07 & 0.14 & 0.807 & 0.121 & 0.488 & 0.072 & 0.360 & 0.057 & 0.134 & 0.020 \\
\hline 140 & 2.3 & & 1.05 & & 1.40 & & 1.60 & & 1.40 & & 1.00 & & 0.65 & & 0.60 & & 0.18 & \\
\hline 150 & 3.0 & & 1.00 & & 1.60 & & 1.80 & & 1.70 & & 1.20 & & 0.85 & & 0.80 & & 0.25 & \\
\hline 160 & 4.0 & & 0.98 & & 1.90 & & 2.40 & & 2.20 & & 1.40 & & 1.10 & & 1.00 & & 0.35 & \\
\hline 170 & 5.5 & & 1.20 & & 2.50 & & 3.00 & & 2.70 & & 1.70 & & 1.30 & & 1.30 & & 0.50 & \\
\hline 180 & 7.0 & & 1.50 & & 4.00 & & 3.80 & & 3.00 & & 2.00 & & 1.50 & & 1.60 & & 0.70 & \\
\hline ICS & 41.5 & 8.5 & 34.0 & 7.0 & 37.0 & 7.6 & 29.3 & 6.0 & 25.6 & 5.3 & 20.8 & 4.3 & 16.1 & 3.3 & 14.1 & 2.9 & 8.07 & 1.66 \\
\hline \multicolumn{19}{|c|}{ (b) Ethanol } \\
\hline 0 & 45.0 & & 160.0 & & 100 & & 70.0 & & 55.0 & & 80.0 & & 100.0 & & 110 & & 220 & \\
\hline 5 & 37.0 & & 85.0 & & 70.0 & & 45.0 & & 40.0 & & 65.0 & & 70.0 & & 72.3 & 10.4 & 88.8 & 13.8 \\
\hline 10 & 30.0 & & 50.0 & & 40.0 & & 26.8 & 3.7 & 27.6 & 3.8 & 33.7 & 4.9 & 39.0 & 5.3 & 37.6 & 5.4 & 28.6 & 4.2 \\
\hline 15 & 22.0 & & 22.8 & 4.21 & 18.9 & 2.6 & 20.0 & & 21.0 & & 20.0 & & 24.0 & & 19.0 & & 12.0 & \\
\hline 20 & 15.0 & & 11.1 & 2.4 & 12.0 & 1.6 & 13.9 & 2.0 & 14.9 & 2.2 & 13.0 & 2.0 & 11.9 & 1.62 & 6.63 & 0.91 & 3.17 & 0.42 \\
\hline 25 & 11.7 & 1.6 & 8.00 & & 7.5 & & 10.0 & & 9.70 & & 7.00 & & 7.00 & & 3.60 & & 1.60 & \\
\hline 30 & 8.30 & 0.87 & 5.47 & 1.26 & 5.59 & 0.85 & 7.16 & 0.95 & 6.48 & 0.86 & 4.69 & 0.65 & 3.14 & 0.45 & 1.60 & 0.242 & 1.09 & 0.15 \\
\hline 40 & 4.86 & 0.58 & 3.45 & 0.59 & 3.04 & 0.46 & 3.69 & 0.55 & 3.26 & 0.45 & 2.20 & 3.31 & 1.60 & 0.23 & 1.04 & 0.156 & 0.525 & 0.072 \\
\hline 50 & 2.86 & 0.53 & 2.49 & 0.58 & 2.30 & 0.35 & 2.44 & 0.34 & 2.18 & 0.32 & 1.65 & 0.23 & 1.27 & 0.18 & 0.630 & 0.088 & 0.308 & 0.042 \\
\hline 70 & 1.69 & 0.30 & 1.80 & 0.37 & 2.10 & 0.28 & 1.73 & 0.23 & 1.435 & 0.199 & 0.960 & 0.141 & 0.554 & 0.080 & 0.284 & 0.040 & 0.124 & 0.016 \\
\hline 90 & 1.60 & 0.31 & 2.06 & 0.43 & 1.53 & 0.22 & 1.25 & 0.19 & 0.961 & 0.130 & 0.646 & 0.088 & 0.353 & 0.052 & 0.192 & 0.029 & 0.079 & 0.011 \\
\hline 110 & 1.88 & 0.25 & 1.71 & 0.36 & 1.17 & 0.16 & 1.58 & 0.23 & 1.16 & 0.17 & 0.719 & 0.101 & 0.423 & 0.064 & 0.188 & 0.026 & 0.130 & 0.019 \\
\hline 130 & 2.17 & 0.33 & 1.41 & 0.27 & 1.28 & 0.19 & 1.82 & 0.24 & 1.36 & 0.18 & 0.957 & 0.135 & 0.633 & 0.096 & 0.366 & 0.050 & 0.190 & 0.028 \\
\hline 140 & 2.5 & & 1.30 & & 1.40 & & 2.00 & & 1.60 & & 1.05 & & 0.73 & & 0.56 & & 0.30 & \\
\hline 150 & 3.0 & & 1.40 & & 1.70 & & 2.40 & & 2.00 & & 1.20 & & 0.90 & & 0.80 & & 0.40 & \\
\hline 160 & 3.5 & & 1.50 & & 2.20 & & 3.00 & & 2.60 & & 1.40 & & 1.10 & & 1.00 & & 0.55 & \\
\hline 170 & 4.2 & & 1.80 & & 3.00 & & 3.70 & & 3.20 & & 1.70 & & 1.40 & & 1.30 & & 0.80 & \\
\hline 180 & 5.0 & & 2.50 & & 4.00 & & 4.50 & & 4.00 & & 2.00 & & 1.70 & & 1.50 & & 1.00 & \\
\hline ICS & 42.7 & 8.8 & 40.3 & 8.3 & 36.6 & 7.5 & 37.7 & 7.7 & 33.3 & 6.8 & 27.4 & 5.6 & 23.8 & 4.9 & 17.5 & 3.6 & 13.0 & 2.7 \\
\hline
\end{tabular}

a closure scheme, described next, in order to properly describe the higher partial waves and improve the differential cross sections in the forward direction for these polar targets.

To account for the long-range character of the dipole interaction we employed a Born correction procedure within the adiabatic approximation to obtain the rotationally unresolved elastic cross section, as is commonly done in treating electron scattering by polar molecules [60]; details of our correction procedure are outlined here. The elastic scattering amplitudes are calculated in the body reference frame (BF) of the molecular target in the SMCPP calculations. In this frame we also calculate the first Born approximation (FBA) to the scattering amplitude for a point-dipole potential with the same orientation and magnitude as the molecular dipole 
used in the SMCPP calculation. These two amplitudes are expanded into partial waves (up to $\ell_{\max }$ ), subtracted from each other $\left(F_{\text {diff }}^{\text {body }}=F_{\mathrm{SMCPP}}-F_{\text {dipole }}\right)$ and transformed into the laboratory frame (giving rise to $F_{\text {diff }}^{\text {lab }}$ ), where the $z$ axis is along the incident wave direction. In the laboratory frame a closed form of the point-dipole amplitude can be obtained in the FBA and added to the resulting amplitude, i.e., $F_{\text {dipole }}^{\text {lab }}$ $+F_{\text {diff }}^{\text {lab }}$ This procedure represents just the replacement of the low partial waves of the full point-dipole amplitude obtained in the FBA with the SMCPP partial waves. The divergence of the forward scattering amplitude can be avoided by making $k_{f}$ slightly different from $k_{i}$. We used $\ell_{\max }=2$ for $E_{0}$ $\leqslant 2 \mathrm{eV}, \ell_{\max }=4$ for $2 \mathrm{eV}<E_{0} \leqslant 5 \mathrm{eV}, \ell_{\max }=5$ for $5 \mathrm{eV}$ $<E_{0} \leqslant 20 \mathrm{eV}$, and $\ell_{\max }=10$ for $E_{0}>20 \mathrm{eV}$. The resulting DCSs resemble the SMCPP DCSs above $30^{\circ}$ and the dipole DCS below $30^{\circ}$, as expected, except at 1 and $2 \mathrm{eV}$, because at these low energies the basis set is not completely adequate.

For the all-electron SMC calculations, methanol and ethanol geometries were optimized within $C_{s}$ point-group symmetry at the level of second-order Möller-Plesset perturbation theory using the $6-31 \mathrm{G}(d)$ basis set [61] and the electronic-structure package GAMESS [62]. Scattering calculations for both methanol and ethanol used the "triple-zeta valence" Gaussian basis set of Dunning [63] together with a supplement of three $d$ polarization functions on $\mathrm{C}$ and $\mathrm{O}$, two $p$ polarization functions on $\mathrm{H}$, one $s$ and one $p$ diffuse function on $\mathrm{C}$ and $\mathrm{O}$, and one diffuse $s$ function on $\mathrm{H}$. The GAMESS default values were used for the exponents and splitting factors of the supplemental functions, and all " 3 " linear combinations of Cartesian $d$ Gaussians were excluded. To obtain a compact configuration space for the representation of polarization effects, the Hartree-Fock virtual orbitals were transformed into MVOs using a +6 ionic core, and singletcoupled single excitations from the Hartree-Fock ground state into low-lying MVOs were used to form the closedchannel space. For methanol, we included excitations from all seven noncore occupied orbitals into the 15 lowest-energy MVOs and coupled those with all 97 virtual orbitals to form doublet configuration state functions, leading to variational spaces of dimension 5030 for $A^{\prime}$ symmetry and 4517 for $A^{\prime \prime}$. For ethanol, we included excitations from the three most tightly bound valence orbitals into the 15 lowest MVOs and excitations from the seven outer valence orbitals into the 25 lowest MVOs, in each case coupled with the 128 lowestenergy MVOs to form doublet configuration state functions describing the closed-channel space; all 146 MVOs were coupled with the Hartree-Fock ground state to form the open-channel space. The resulting variational spaces were of dimension $13372\left(A^{\prime}\right)$ and $12519\left(A^{\prime \prime}\right)$.

Long-range scattering by the electric-dipole potential was accounted for in the all-electron SMC calculations via a Born-dipole correction procedure very similar to that used in the SMCPP calculations described above. The body-frame SMC scattering amplitude $f^{\mathrm{SMC}}\left(\mathbf{k}_{\text {in }}, \mathbf{k}_{\text {out }}\right)$ was first expanded into the half-partial-wave form $f^{\mathrm{SMC}}\left(\mathbf{k}_{\text {in }}, \ell_{\text {out }}, m_{\text {out }}\right)$ for each direction of incidence $\mathbf{k}_{\text {in }}$ and then rotated into the laboratory frame ( $\mathbf{k}_{\text {in }}$ parallel to the $z$ axis). The same rotation was applied to the molecular dipole vector obtained from the Hartree-Fock calculation (magnitude 1.84 D for methanol and $1.74 \mathrm{D}$ for ethanol), and the first Born approximation to the point-dipole scattering amplitude $f^{\mathrm{BD}}$ for the rotated dipole was evaluated analytically in the same half-expanded representation and subtracted from $f^{\mathrm{SMC}}$. The resulting difference amplitude, up to a specified $\ell_{\max }$ (taken to be 5 for both methanol and ethanol), was then added to the planewave, body-frame Born amplitude for the rotated point dipole, $f^{\mathrm{BD}}\left(k \mathbf{z}, \mathbf{k}_{\text {out }}\right)$, where $\mathbf{z}$ is a unit vector in the $+z$ direction and $\mathbf{k}_{\text {out }}$ is chosen to form the desired scattering angle with the $z$ axis, and the squared absolute value of the combined amplitude was integrated analytically over the azimuthal angle of $\mathbf{k}_{\text {out }}$. The procedure was repeated for each $\mathbf{k}_{\text {in }}$ and the results averaged with appropriate weights (the $\mathbf{k}_{\text {in }}$ directions being quadrature points on the sphere). Note that the combination of this average with the integration over $\mathbf{k}_{\text {out }}$ azimuths constitutes the full average over molecular orientations that is required for comparison with gas-phase measurements. Although different in implementation details, our Born-dipole correction procedure relies on the two essential ideas of earlier successful procedures [64,65], namely that the high-level calculation is used to correct the low partial waves of the Born-dipole approximation and that, in contrast to the procedure followed in other recent work $[10,11]$, the correction is applied to the amplitude and not to the cross section.

\section{RESULTS}

The results of our measurements are shown in Table II. The methanol DCSs are compared to the results of calculations in Fig. 1 and those for ethanol in Fig. 2. The SMCPP results for methanol include polarization effects up to $10 \mathrm{eV}$, while the results above $10 \mathrm{eV}$ were obtained in the staticexchange (SE) approximation. The results at $50 \mathrm{eV}$ and 100 $\mathrm{eV}$ were obtained only with the SMCPP method.

The agreement between the measured and calculated values for $\mathrm{CH}_{3} \mathrm{OH}$ is qualitatively excellent at all energies. The largest differences between experiment and theory occur at lower impact energies, where the differences between the two calculations are also largest; this may reflect limitations in the treatment of polarization but may also be due to differences in the partial-wave cutoff for the Born-dipole corrections. The dip at small angle in the all-electron cross section may be an artifact of the Born-correction procedure; the uncorrected DCS lacks this feature. Moreover, the measurements are most difficult, and therefore most uncertain, at low energies. Clearly, however, both experiment and theory indicate that scattering by the dipole potential becomes very important at low $E_{0}$ values, e.g., at $5 \mathrm{eV}$ or below, as may be seen by comparing the results with and without dipole corrections. At $E_{0}=10,15,20,30$, and $50 \mathrm{eV}$, agreement between theory and experiment is excellent. The measured cross sections are smaller than the calculated values for large angles at $E_{0}=100 \mathrm{eV}$ and rise more steeply at small $\theta$ values. Similar observations may be made concerning the DCS for $\mathrm{C}_{2} \mathrm{H}_{5} \mathrm{OH}$ shown in Fig. 2. However, at higher $E_{0}$ values, the differences between theory and experiment are larger for $\mathrm{C}_{2} \mathrm{H}_{5} \mathrm{OH}$ than for $\mathrm{CH}_{3} \mathrm{OH}$.

Our calculated integral elastic scattering cross sections are shown for methanol and ethanol in Figs. 3 and 4, respec- 

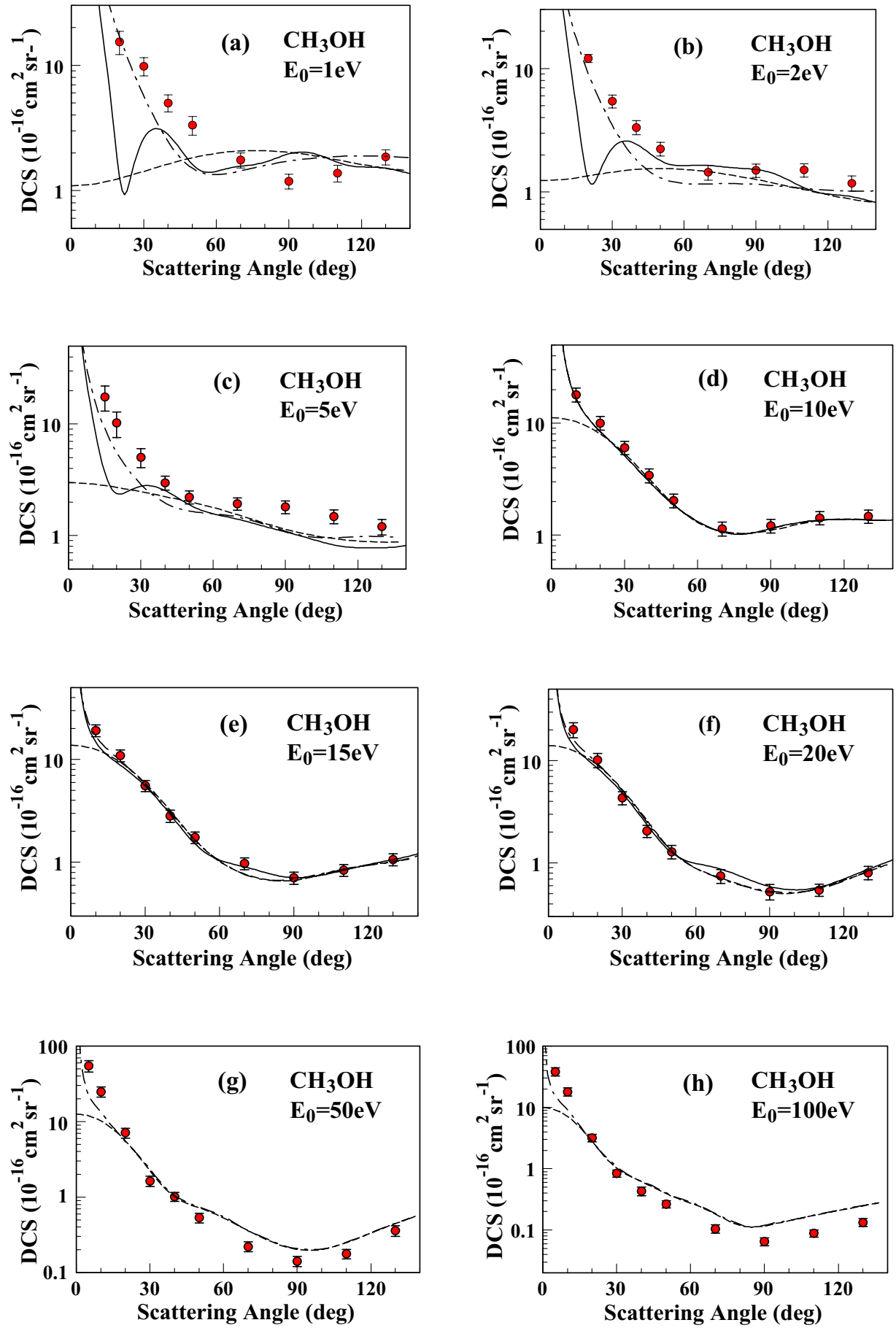

tively. The computed SEP cross sections were obtained by integrating the computed DCS and therefore include the Born correction. The SE cross section shown in this figure was obtained without the Born correction. Since both SMC and SMCPP SE cross sections agree with each other we chose to show only the SMC results. Also shown in Fig. 3 are the calculated elastic cross sections for $\mathrm{CH}_{3} \mathrm{OH}$ of Bouchiha et al. [10] using the $R$-matrix method and the measured total electron scattering cross section of Szmytkowski and Krzysztofowicz [24]. The results of Bouchiha et al. [10] are those obtained without the dipole-Born correction; the correction procedure used in Ref. [10], in which cross sections rather than amplitudes are added, leads to a cross sec- tion far larger than the measured integral cross section at all energies. As may be seen in Fig. 3, the all-electron and the SMCPP cross sections with polarization effects included are very similar above $7 \mathrm{eV}$ and both are in reasonable agreement with the measured total cross section from 12 to $20 \mathrm{eV}$. However, the calculations place the broad peak in the elastic cross section at about $10 \mathrm{eV}$, while the peak in the total cross section lies near $8 \mathrm{eV}$. Moreover, the calculated values differ from the measured total cross section near the low-energy minimum. The present experimental ICS results are markedly higher than all the measured total cross sections and are closest to those of [22]. We cannot attribute this difference to any known error, especially as our ICSs agree with the total 

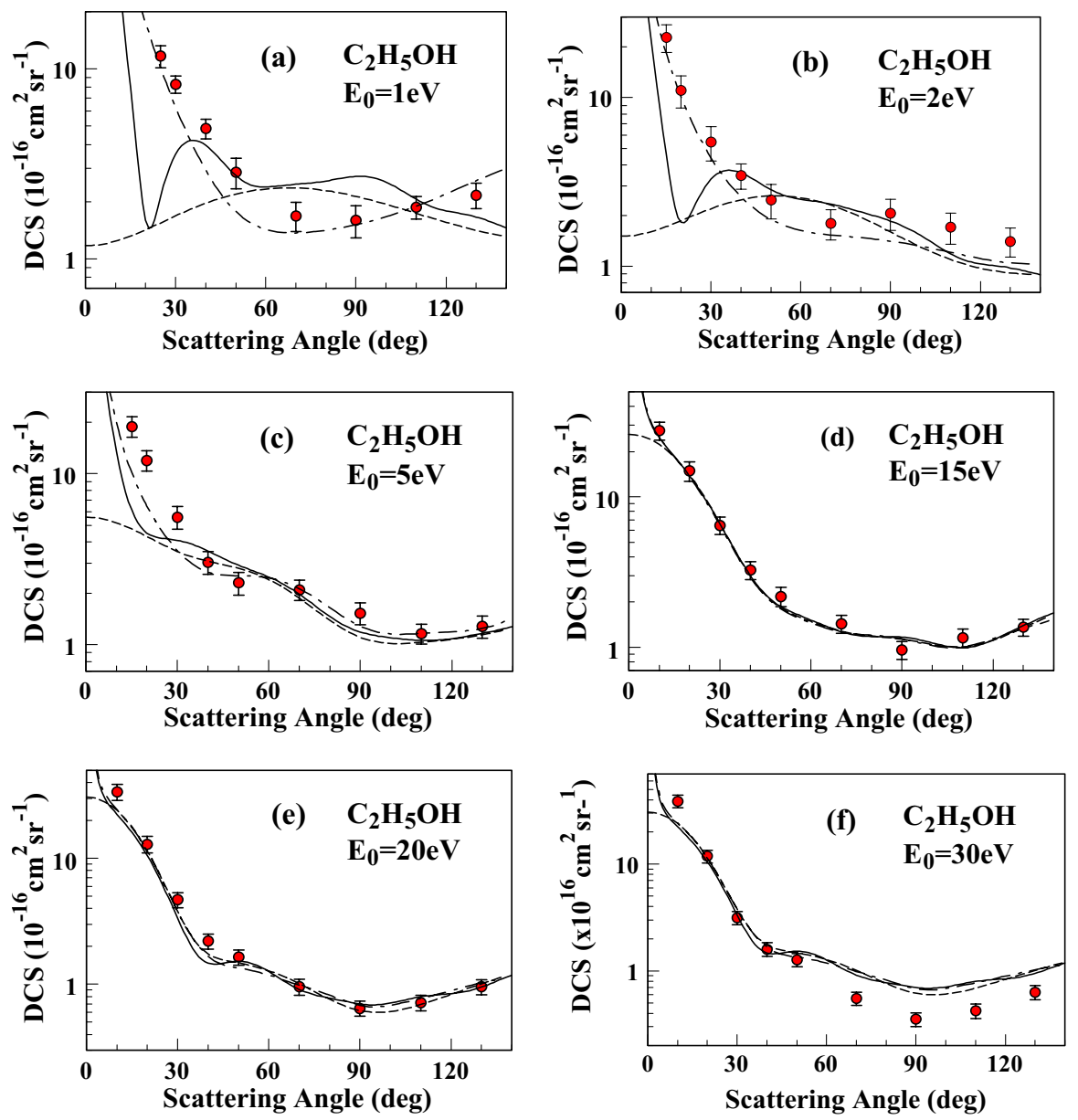

FIG. 2. (Color online) Differential cross sections for elastic electron scattering from ethanol. : present experiment (1 standard deviation error bars); -: allelectron Schwinger multichannel calculation; - - : pseudopotential Schwinger multichannel calculation; and ----: pseudopotential Schwinger multichannel calculation without the Born-dipole correction. At $50 \mathrm{eV}$ and $100 \mathrm{eV}$ the solid line corresponds to the SMCPP results with Born.
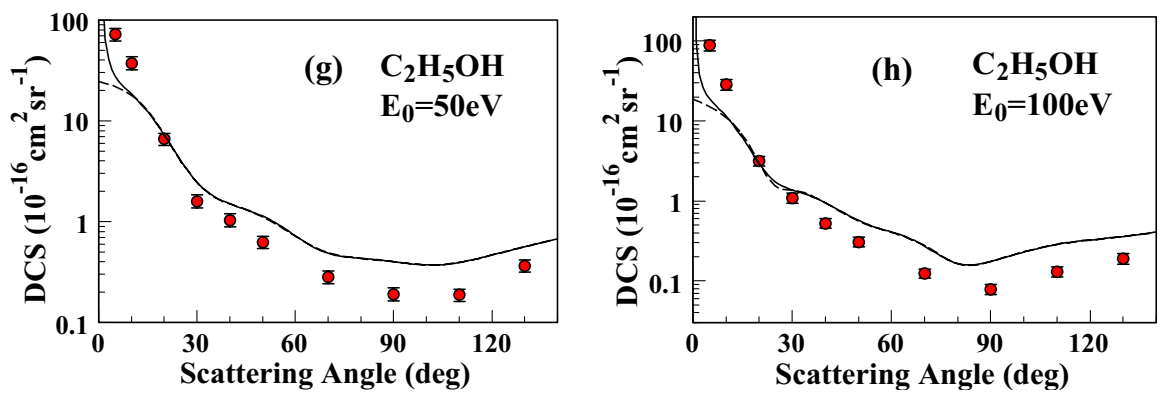

cross sections at the higher energies. We note that in the range of these cross section measurements, the measured total cross sections of Szmytkowski and Krzysztofowicz [24] are in good agreement with earlier work of Sueoka et al. [23], but somewhat lower than those of [22]. We also note that our calculated ICS results agree in magnitude even at low energies with the present experimental ICSs. Consequently, the origin of this disagreement is unclear at present. The $R$-matrix results [10], which place the broad maximum still higher in energy, at about $12.5 \mathrm{eV}$, are very similar to our static-exchange results, suggesting an incomplete representation of polarization effects. In terms of comparisons with total cross sections, the contribution of inelastic scattering channels (vibrational and electronic excitation) at incident energies at or below $20 \mathrm{eV}$ is estimated to be less than $10 \%$ of the elastic integral cross section from separate measurements of the elastic plus inelastic energy loss spectrum of ethanol at $20 \mathrm{eV}$, where it was observed that the vibrational excitation did not exceed $5 \%$ of the elastic scattering.

None of the cross sections in Fig. 3 show any evidence of the low-energy $\mathrm{OH} \sigma^{*}$ shape resonance recently postulated by Ibănescu et al. [31] that gives rise to a peak near $3 \mathrm{eV}$ in the dissociative attachment cross section, nor is there clear evidence of the attachment peaks seen near 6.5, 8, and $10 \mathrm{eV}$ [26-29,31]. The latter peaks are assigned [29] to electronic Feshbach resonances that might be very weak and/or narrow in the elastic channel and in any case would probably not be seen in calculations of the present type. As for the $3 \mathrm{eV}$ feature, Ibănescu et al. [31] note that the peak seen in their attachment spectrum may be broader in other channels, whereas in dissociative attachment it is sharpened on the low-energy side by the thermodynamic threshold for dissociation and on the high-energy side by the increasing autodetachment rate; indeed, in the $\mathrm{O}-\mathrm{H}$ stretch vibrational exci- 


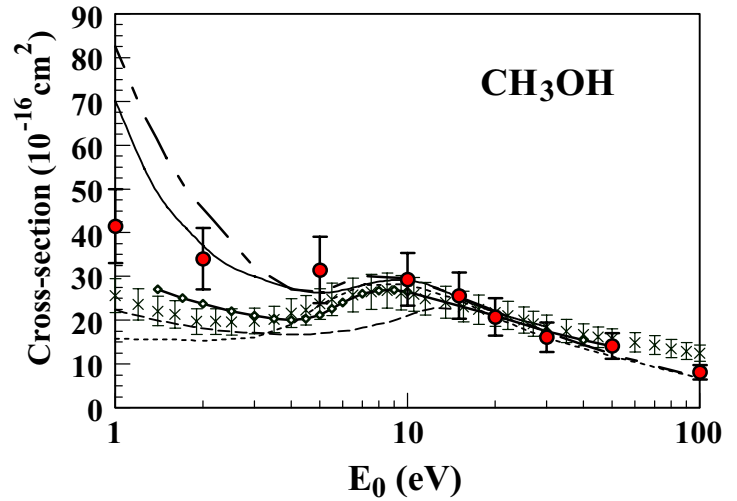

FIG. 3. (Color online) Integral elastic scattering cross sections for $\mathrm{CH}_{3} \mathrm{OH}$. Legend: 0 : present experiment; —- - SMCPP SEP; - : SMC SEP; ---- (short dashes): SMC SE which is similar to SMCPP SE; and ---- (long dashes): $R$-matrix ICSs of Bouchiha et al. (without Born correction) [10]. $\times$ Total cross section measurements of Szmytkowski and Krzysztofowicz [24] and $-\downarrow$ of Schmieder [22]. The ICSs of [23] are in very good agreement with [24] and are not shown here to avoid congestion in the graph. One standard deviation error bars are shown.

tation cross section, it appears as a broad feature peaking at or near $0 \mathrm{eV}$ [29].

Many of the above remarks on methanol apply to the calculated elastic cross sections for ethanol shown in Fig. 4, although in this case we have no experimental total cross section or other calculated cross sections for comparison. Again the two SMC calculations agree quite well with each other, but neither shows evidence of the resonance peaks seen in the dissociative attachment spectra [28,31,33], which may be too weak and/or broad to be seen clearly in the elastic channel.

\section{CONCLUSIONS}

We have used the relative flow technique with an aperture source [43], coupled with a moveable gas target method [47], to carry out the first measurements of DCSs for the two polyatomic molecules, methanol and ethanol, whose gas-

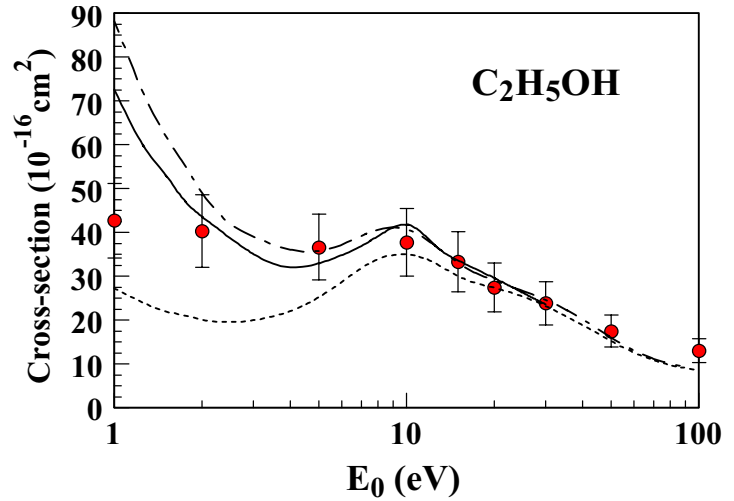

FIG. 4. (Color online) Integral elastic scattering cross sections for $\mathrm{C}_{2} \mathrm{H}_{5} \mathrm{OH}$. Legend is the same as Fig. 3 except that ----- (short dashes) are from the SMCPP SE which is similar to SMC SE.

kinetic molecular diameters are not well-established. Comparison of these measured cross sections with results of calculations is encouraging. We are presently extending our measurements and calculations to other alcohols, namely propanol $\left(\mathrm{C}_{3} \mathrm{H}_{7} \mathrm{OH}\right)$ and butanol $\left(\mathrm{C}_{4} \mathrm{H}_{9} \mathrm{OH}\right)$.

\section{ACKNOWLEDGMENTS}

This work was funded by the National Science Foundation through Grant No. PHY 0653452, under a collaborative program with CNPq-Brazil. We acknowledge work on the moveable source control electronics by Dr. James Gregory Childers and Narciso Guzman. Work by V.M. and C.W. was also supported by the Chemical Sciences, Geosciences and Biosciences Division, Office of Basic Energy Sciences, Office of Science, U.S. Department of Energy, and made use of the Jet Propulsion Laboratory's Supercomputing and Visualization Facility. M.C.A.L., H.S., R.F. da C., L.G.F., M.A.P.L., and M.H.F.B. acknowledge support from Conselho Nacional de Desenvolvimento Científico e Tecnológico (CNPq), Fundação de Amparo à Pesquisa do Estado de São Paulo (FAPESP), Fundação Araucária (Paraná State Agency), Finep (under project CT-Infra), and Coordenação de Aperfeiçoamento de Pessoal de Nível Superior (CAPES). Some of the computer calculations in Brazil were performed at the CENAPAD-SP.
[1] M. J. Brunger and S. J. Buckman, Phys. Rep. 357, 215 (2002).

[2] C. T. Whelan and N. J. Mason, Electron Scattering from Atoms, Molecules, Nuclei and Bulk Matter (Kluwer Academic/ Plenum, New York, 2005).

[3] G. G. Lister, J. E. Lawler, W. P. Lapatovich, and V. A. Godyak, Rev. Mod. Phys. 76, 541 (2004).

[4] D. J. Strickland, J. L. Lean, R. R. Meier, A. B. Christensen, L. J. Paxton, D. Morrison, J. D. Craven, R. L. Walterscheid, D. L. Judge, and D. R. McMullin, Geophys. Res. Lett. 31, L03801 (2004).

[5] B. Boudaïffa, P. Cloutier, D. Hunting, M. A. Huels, and L. Sanche, Science 287, 1658 (2000).

[6] B. Block, P. Möser, and W. Hentschel, Opt. Eng. 36, 1183 (1997).
[7] S. Y. Liao, D. M. Jiang, Q. Cheng, Z. H. Huang, and K. Zeng, Energy Fuels 20, 84 (2006).

[8] See, e.g., B. Sulik, Radiat. Phys. Chem. 76, 483 (2007).

[9] P. C. Stangeby, The Plasma Boundary of Magnetic Fusion Devices (Institute of Physics, Philadelphia, 2000).

[10] D. Bouchiha, J. D. Gorfinkiel, L. G. Caron, and L. Sanche, J. Phys. B 40, 1259 (2007).

[11] D. Bouchiha, J. D. Gorfinkiel, L. G. Caron, and L. Sanche, J. Phys. B 39, 975 (2006).

[12] S. Tonzani and C. H. Greene, J. Chem. Phys. 124, 054312 (2005).

[13] S. Tonzani and C. H. Greene, J. Chem. Phys. 125, 094504 (2006).

[14] C. Winstead and V. McKoy, J. Chem. Phys. 125, 074302 
(2006)

[15] C. Winstead and V. McKoy, J. Chem. Phys. 125, 174304 (2006).

[16] C. Winstead and V. McKoy, J. Chem. Phys. 125, 244302 (2006).

[17] C. Winstead, V. McKoy, and S. d'A. Sanchez, J. Chem. Phys. 127, 085105 (2007).

[18] M. H. F. Bettega, Phys. Rev. A 74, 054701 (2006).

[19] M. H. F. Bettega and M. A. P. Lima, J. Chem. Phys. 126, 194317 (2007).

[20] R. F. da Costa, M. H. F. Bettega, and M. A. P. Lima, Phys. Rev. A 77, 012717 (2008).

[21] D. Mathur and J. B. Hasted, Chem. Phys. Lett. 34, 90 (1975).

[22] F. Schmieder, Z. Elektrochem. Angew. Phys. Chem. 36, 700 (1930).

[23] O. Sueoka, Y. Katayama, and S. Mori, At. Coll. Res. Japan, Prog. Rep. 11, 17 (1985).

[24] C. Szmytkowski and A. M. Krzysztofowicz, J. Phys. B 28, 4291 (1995).

[25] L. von Trepka and H. Neuert, Z. Naturforsch. A 18, 1295 (1963).

[26] H. Kühn, H.-P. Fenzlaff, and E. Illenberger, J. Chem. Phys. 88, 7453 (1988).

[27] M. G. Curtis and I. C. Walker, J. Chem. Soc., Faraday Trans. 88, 2805 (1992).

[28] V. S. Prabhudesai, A. H. Kelkar, D. Nandi, and E. Krishnakumar, Phys. Rev. Lett. 95, 143202 (2005).

[29] T. Skalický and M. Allan, J. Phys. B 37, 48 (2004).

[30] L. Parenteau, J. P. Jay-Gerin, and L. Sanche, J. Phys. Chem. 98, 10277 (1994).

[31] B. C. Ibănescu, O. May, A. Monney, and M. Allan, Phys. Chem. Chem. Phys. 9, 3163 (2007).

[32] A. T. Wen, M. Michaud, and L. Sanche, Phys. Rev. A 54, 4162 (1996).

[33] M. Orzol, I. Martin, J. Kocisek, I. Dabkowska, J. Langer, and E. Illenberger, Phys. Chem. Chem. Phys. 9, 3424 (2007).

[34] S. K. Srivastava, A. Chutjian, and S. Trajmar, J. Chem. Phys. 63, 2659 (1975).

[35] S. Trajmar, D. F. Register, and A. Chutjian, Phys. Rep. 97, 219 (1983).

[36] R. K. Nesbet, Phys. Rev. A 20, 58 (1979).

[37] D. F. Register, S. Trajmar, and S. K. Srivastava, Phys. Rev. A 21, 1134 (1980).

[38] A. R. Milosavljević, A. Giuliani, D. Šević, M. J. HubinFranskin, and B. P. Marinković, Eur. Phys. J. D 35, 411 (2005).

[39] M. Dampc, A. R. Milosavljević, I. Linert, B. P. Marinković, and M. Zubek, Phys. Rev. A 75, 042710 (2007).

[40] C. J. Colyer, V. Vizcaino, J. P. Sullivan, M. J. Brunger, and S. J. Buckman, New J. Phys. 9, 41 (2007).

[41] M. Allan, J. Phys. B 40, 3531 (2007).
[42] Landolt-Börnstein, Zahlenwerte und Funktionen, Bd I, Atom und Molekularphysik, 1, Atome und Ionen, edited by A. Eucken (Springer-Verlag, Berlin, 1950).

[43] M. A. Khakoo, K. Keane, C. Campbell, N. Guzman, and K. Hazlett, J. Phys. B 40, 3601 (2007).

[44] C. Winstead and V. McKoy, Comput. Phys. Commun. 128, 386 (2000).

[45] M. H. F. Bettega, L. G. Ferreira, and M. A. P. Lima, Phys. Rev. A 47, 1111 (1993).

[46] M. A. Khakoo, C. E. Beckmann, S. Trajmar, and G. Csanak, J. Phys. B 27, 3159 (1994).

[47] M. Hughes, K. E. James, Jr., J. G. Childers, and M. A. Khakoo, Meas. Sci. Technol. 14, 841 (2003).

[48] E. Schow, K. Hazlett, J. G. Childers, C. Medina, G. Vitug, I. Bray, D. V. Fursa, and M. A. Khakoo, Phys. Rev. A 72, 062717 (2005).

[49] J. G. Childers, K. E. James, M. Hughes, I. Bray, M. Baertschy, and M. A. Khakoo, Phys. Rev. A 68, 030702 (2003).

[50] M. A. Khakoo, T. Jayaweera, S. Wang, and S. Trajmar, J. Phys. B 26, 4 (1993).

[51] T. Sagara and L. Boesten, J. Phys. B 31, 3455 (1998).

[52] F. Rugamas, D. Roundy, G. Mikaelian, G. Vitug, M. Rudner, J. Shih, D. Smith, J. Segura, and M. A. Khakoo, Meas. Sci. Technol. 11, 1750 (2000).

[53] J. H. Brunt, G. C. King, and F. H. Read, J. Phys. B 10, 1289 (1977).

[54] National Institute of Standards and Technology, Standard Reference Data at URL: http://srdata.nist.gov/cccbdb/default.html

[55] M. H. F. Bettega, A. P. P. Natalense, M. A. P. Lima, and L. G. Ferreira, Int. J. Quantum Chem. 60, 821 (1996).

[56] W. J. Hunt and W. A. Goddard, Chem. Phys. Lett. 3, 414 (1969).

[57] C. W. Bauschlicher, J. Chem. Phys. 72, 880 (1980).

[58] T. N. Rescigno, C. W. McCurdy, and B. I. Schneider, Phys. Rev. Lett. 63, 248 (1989).

[59] CRC Handbook of Chemistry and Physics, 58th ed., edited by R. C. Weast (CRC Press, Boca Raton, FL, 1977).

[60] Y. Okamoto, K. Onda, and Y. Itikawa, J. Phys. B 26, 745 (1993).

[61] R. Ditchfield, W. J. Hehre, and J. A. Pople, J. Chem. Phys. 54, 724 (1971); W. J. Hehre, R. Ditchfield, and J. A. Pople, ibid. 56, 2257 (1972); P. C. Hariharan and J. A. Pople, Theor. Chim. Acta 28, 213 (1973).

[62] M. W. Schmidt, K. K. Baldridge, J. A. Boatz, S. T. Elbert, M. S. Gordon, J. H. Jensen, S. Koseki, N. Matsunaga, K. A. Nguyen, S. J. Su, T. L. Windus, M. Dupuis, and J. A. Montgomery, J. Comput. Chem. 14, 1347 (1993).

[63] T. H. Dunning, Jr., J. Chem. Phys. 55, 716 (1971).

[64] A. W. Fliflet and V. McKoy, Phys. Rev. A 21, 1863 (1980).

[65] T. N. Rescigno and B. I. Schneider, Phys. Rev. A 45, 2894 (1992), and references therein. 